\title{
PEDIATRIC SURGICAL ONCOLOGY IN BANGLADESH: INCIDENCE AND PREVALENCE (GLOBAL \& BANGLADESH)
}

Pediatric surgical oncology is a relatively new and rapidly evolving field. Childhood neoplasia is generally not a public health priority in most developing countries. Although it is rare, pediatric cancer is a leading cause of childhood death in developed countries such as the United States. ${ }^{1}$ In the 1960s, almost $25 \%$ of global cancer burden was diagnosed in low-income and lower-middle-income countries. ${ }^{1}$ In 2010 , nearly $55 \%$ of the global cancer burden was found in these countries. ${ }^{1}$ According to estimates from the International Agency for Research on Cancer (IARC), there were 12.7 million new cancer cases in 2008 worldwide, of which 5.6 million occurred in developed countries and 7.1 million in developing countries. ${ }^{1}$ Total cancer deaths in 2008 were 7.6 million (about 21,000 cancer deaths a day), 2.8 million in developed countries and 4.8 million in developing countries. ${ }^{1}$ By 2030 , the global burden is expected to grow to 21.4 million new cancer cases and 13.2 million cancer deaths. ${ }^{1,2}$ Almost 9 million (about $70 \%$ ) of these deaths will be in developing countries. ${ }^{2,3}$ By 2050 , at the present growth rate, the chances of contracting cancer in their lifetime for the people living in developing countries will be $50-60 \% .{ }^{3}$ Worldwide, approximately 10 million people are diagnosed with cancer annually and more than 6 million die of the disease every year; currently, over 22 million people in the world are cancer patients. ${ }^{4,5}$ The cancer rate will increase from 650,000 to 2.2 million per year. ${ }^{4,5}$ In developed countries like United States, 11,600 new cases of pediatric malignancies are expected to be diagnosed in children aged 0-14 years in 2013. ${ }^{5}$ In 2008, GLOBOCAN has estimated that about 1, 48,000 malignancies in children aged $0-14$ years occurred in less developed regions like Asia excluding Japan, Africa etc. having a population of 5.5 billion. ${ }^{1,5,6}$ There is no national population based cancer registry in Bangladesh but using worldwide incidence rates, there are 13 to 15 lakh cancer patients with about 2 lakh patients newly-diagnosed with cancer each year. ${ }^{4}$ Using the same incidence rates of between 80 and $150 /$ million children we would expect 6000-9000 new cases/year in Bangladesh. ${ }^{4}$ The greatest variation in incidence of pediatric malignancies occurs in comparisons of high-income to low-income countries and may derive from incomplete ascertainment of pediatric malignancy occurrence, different risk, or differences in risk among different ethnic or racial population subgroups. ${ }^{7,8}$

\section{Approach:}

Up to the early $20^{\text {th }}$ century the only modality for the treatment of childhood solid tumors was radical resection. By 1920's, radiation therapy (RT) was added and much later, toward the mid-20 $20^{\text {th }}$ century, single drug chemotherapy was introduced. The first being actinomycin $D$ for Wilms' tumor. These single drug therapies were gradually replaced by multi-drug combination regimen, which demonstrated improved response rates. By the 1970's multimodal therapy including multi-drug chemotherapy, surgical resection and RT was shown to have even better cure rates in Wilms' tumor. Subsequently, similar multimodal protocols have been adopted for other tumors such as neuroblastoma, rhabdomyosarcoma, Ewing's sarcoma, malignant germ cell tumors, and other solid tumors. By 1990's, dose-intensive regime was introduced with other refinements in the dosage and the mode of administration. Simultaneous improvements in RT have led to decrease in the radiation dosage and more targeted $\mathrm{RT}$, thereby decreasing the long-term morbidities. In the 
$21^{\text {st }}$ century, as the biologic pathways for childhood cancers became better understood, the research is mainly focused on less cytotoxic biologic therapies such as anti-angiogenesis agents, growth factor receptor inhibitors, signal transduction inhibitors, targeted antibodies and immunotherapy. ${ }^{9}$ With all the advances in the treatment of pediatric solid tumors, very high long-term survival of $>85-90 \%$ has now been achieved in USA and other developed countries. ${ }^{10}$ In developing countries, the estimated overall five-year survival rates for childhood cancer were as low as 40$60 \%$ in Egypt, Honduras, and Venezuela; $30 \%$ in Morocco; and 5-10\% in Bangladesh, the Philippines, Senegal, Tanzania, and Vietnam. ${ }^{11}$ This is why the aim of treatment for childhood cancers in developed countries, now is "childhood cancer is curable." 9 This is the success story of the multi-drug multimodal therapy as has been achieved by some of the well conducted multi-center co-operative trials like the SIOP, NWTSG, COG study for Wilms' tumor and the SIOPEL studies for liver tumors. ${ }^{9}$

Pediatric solid tumors are a heterogeneous group with different malignancies affecting different organs, each having its own staging system, prognostic factors and varied chemotherapy protocols. Similarly, a number of pediatric solid tumor cases are operated upon by adult surgeons who tend to extend their knowledge of adult solid tumors on to the management of pediatric tumors. Almost all pediatric solid tumors need adjuvant chemotherapy except rare situations like stages I and II Wilms' tumor and neuroblastoma, stage I endodermal sinus tumor of the testis. At the other end, there are some tumors, like adrenal carcinomas, where surgical resection is the only therapy that would result in survival and chemotherapy has very little role to play. This is why, most pediatric surgeons in Bangladesh should be trained to administer chemotherapy for the solid tumors that they operate upon or else just for the want of pediatric oncologists the overall treatment of the children with solid tumors will remain incomplete. Most solid tumor protocols are easy to follow and have minimal side effects and complications if one follows the general guidelines of dilution, rate of administration of fluids and the interval of administration. ${ }^{9}$ The surgeon, on his part, can't alter the tumor biology but can ensure gross complete removal, avoid upstaging the tumors by avoiding spill and incomplete resections, avoid the unnecessary resection of contiguous organs and help appropriately staging the tumor. This will in turn ensure appropriate therapy so as to give the maximum benefit to the patient with minimum morbidity. For doing a proper job, the pediatric surgeon should have knowledge about the natural history, biology and behavior of the tumor, knowledge about diagnostic procedures and staging. $\mathrm{He} / \mathrm{she}$ should also have the knowledge of proper tissue handling of the biopsy or resected specimens so as not to ensure proper pathological and molecular studies.

\section{Where are we?}

Bangladesh is a developing country that is facing many challenges, especially in the health sector. It is unfortunate that few tertiary care centers in Bangladesh that have both pediatric oncology and pediatric surgery have not still got together to manage the patient in a multidisciplinary team approach. Multidisciplinary approach is a must and consultation upfront, among the pediatric surgeons, pediatric oncologists and radiation oncologists can easily draw a road map for the treatment of every individual child with a specific malignancy. Surgeons should restrain their urge to resect every mass that they come across as an initial treatment. These results in failed resections, unnecessary resections of adjoining organs and tumor spill with subsequent upstaging of the disease. There is a very clear understanding now that for most pediatric solid tumors, neoadjuvant chemotherapy (preoperative chemotherapy) has a definite advantage in decreasing the risk of major resections. It must be realized that now the cure rates in pediatric solid tumors are very high, and any complication may decrease the subsequent qualityof-life for the patient for the next 50-60 years.

\section{Challenges:}

The high cost of treatment, lack of adequate radiation facilities, dearth of trained manpower, and lack of awareness within the community are the main challenges impeding cancer care in Bangladesh. Unavailability of trained radiotherapist is also a significant barrier in the development of radiation treatment facilities in our country. ${ }^{12}$ Further, there is hardly any data available for the treatment and the outcomes of pediatric solid tumors from Bangladesh. Even though, many centers have treated a large number of patients, there is an extreme paucity of data.

\section{Conclusion and Recommendation:}

Most of the childhood tumors are surgically curable except Hodgkin's lymphoma, if it is diagnosed early. 
Although the treatment facilities are not up to the mark due to poor resources but still we are trying to give the truthful management. We would also like to urge centers to maintain good data, analyze them, present and publish these. That is the only way forward to understand the disease behavior in our country and modify protocols available from the developed world; to obtain the best possible results in the resourcechallenged setting. Availability of such single center outcome results will enable and encourage us to establish multi center trials, especially designed for our country that will keep in mind the various constraints.

If government, as well as private sectors does launch programs on pediatric tumors to increase consciousness among mass people and also increase finance to train the health care personnel and treatment amenities. So that we can lessen the burden of childhood tumors and can give the child a better way of living. With the current trend of development at both government and private sectors, it is assumed that the scenario of cancer management will move to a much more impressive state in the near future.

Dr. Kaniz Hasina

Associate Professor

Department of Pediatric Surgery

Dhaka Medical College \& Hospital

E-mail:kanizhasina@gmail.com

\section{References}

1. Global Malignancy Facts \& Figures 2nd Edition. American Malignancy Society. 2008. http:// www.malignancy.org/research/malignancy factsfigures/globalmalignancyfactsfigures/globalfacts-figures-2nd-ed

2. GLOBOCAN 2008: Cancer Incidence and Mortality Worldwide. website: http://globocan. iarc.fr

3. Noronha V, Tsomo U, Jamshed A, Hai MA, Wattegama S, Baral RP, et al. A fresh look at oncology facts on south central Asia and SAARC countries. South Asian J Cancer. 2012; 1:14. [PMC free article][PubMed]

4. Ferlay J, Bray F, Pisani P, Parkin MD. IARC Cancer Base No. 5, Version 2.0. Lyon:
International Agency for Cancer Research Press (IARC); 2004. Globocan 2002: Cancer incidence, mortality and prevalence worldwide.

5. Ferlay J, Shin HR, Bray F, Forman D, Mathers C, Parkin DM. Estimates of worldwide burden of cancer in 2008: GLOBOCAN 2008. Int J Cancer. 2010;127:2893-917. [PubMed]

6. Kumar P, Bhattacharyya GS, Dattatreya S, Malhotra $\mathrm{H}$. Tackling the cancer Tsunami. Indian J Cancer.2009; 46:1-4. [PubMed]

7. Magrath I, Steliarova-Foucher E, Epelman S, Ribeiro RC, Harif M, Li CK, Kebudi R, Macfarlane SD, Howard SC. Pediatric malignancy in lowincome and middle-income countries. Lancet Oncol. 2013 Mar; 14(3):e104-16.

8. Scott C. Howard, Monika L. Metzger, Judith A. Wilimas, Yuri Quintana, Ching-Hon Pui, Leslie L. Robison and Raul C. Ribeiro. Childhood malignancy epidemiology in low-income countries. Malignancy, 2007, Volume 112, Issue 3, pages 461-472.

9. Sandeep Agarwala. Pediatric surgical oncology in India. J Indian Assoc Pediatr Surg. 2014 OctDec; 19(4): 187-188.

10. Altekruse SF, Kosary CL, Krapcho M, Neyman N, Aminou R, Waldron W, Ruhl J, Howlader N, Tatalovich Z, Cho H, Mariotto A, Eisner MP, Lewis DR, Cronin K, Chen HS, Feuer EJ, Stinchcomb DG, Edwards BK (eds). SEER Cancer Statistics Review, 1975-2007, National Cancer Institute. Bethesda, MD, http://seer.cancer.gov/csr/ 1975_2007/, based on November 2009 SEER data submission, posted to the SEER web site, 2010.

11. Ribeiro RC, Steliarova-Foucher E, Magrath I, et al. Baseline status of paediatric oncology care in ten low-income or mid-income countries receiving My Child Matters support: a descriptive study. Lancet Oncol. Aug 2008; 9(8):721-729.

12. A.F.M. Kamal Uddin, Zohora Jameela Khan, Johirul Islam, and AM Mahmud. Cancer care scenario in Bangladesh. South Asian J Cancer. 2013 Apr-Jun; 2(2): 102-104. 